\title{
Kounal of Biomedicine
}

2017; 2: 134-139. doi: 10.7150/jbm.18949

Review

\section{Role of CXCL12/CXCR4-Mediated Circulating Fibrocytes in Pulmonary Fibrosis}

\author{
Linshen Xie, Liqiang Zhao ${ }^{\bowtie}$ \\ West China School of Public Health /No.4 West China Hospital, Sichuan University, China \\ $\triangle$ Corresponding author: Liqiang Zhao, hxsy_sc@163.com \\ ( $)$ Ivyspring International Publisher. This is an open access article distributed under the terms of the Creative Commons Attribution (CC BY-NC) license \\ (https://creativecommons.org/licenses/by-nc/4.0/). See http://ivyspring.com/terms for full terms and conditions.
}

Received: 2017.02.07; Accepted: 2017.09.12; Published: 2017.10.02

\begin{abstract}
Pulmonary fibrosis is characterized by excessive deposition of extracellular matrix (ECM) and remodeling of the lung architecture, with clinically irreversible loss of lung function. The exact molecular and cellular mechanisms of pulmonary fibrosis are complicated. Many types of cells are involved in the pathogenic processes. The chemokine (C-X-C motif) ligand 12 (CXCL12) can attract circulating fibrocytes trafficking into lungs via chemokine (C-X-C motif) receptor 4 (CXCR4) to promote tissue repair or impertinently worsen fibrotic lesions. In this review, we briefly summarize the existing experimental and clinical findings about fibrocytes in pulmonary fibrosis and discuss the potential therapeutic target CXCL12/CXCR4 biological axis.
\end{abstract}

Key words: Pulmonary fibrosis; Fibrocytes; CXCL12/CXCR4.

\section{Introduction}

Pulmonary fibrosis is the common pathway of a number of lung disease that are characterized by alveolar epithelial injury, fibroblasts and myofibroblast proliferation, collagen overproduction, excessive extracellular matrix (ECM) accumulation, and pulmonary neovascularization ${ }^{1}$. Pulmonary fibrosis could be induced by a lot of factors such as infection, medicine, radiation, chemical agent, organic or inorganic dust and autoimmune diseases (e.g. scleroderma) ${ }^{2}$. Since the pathogenic mechanisms of pulmonary fibrosis are complicated, resulting in the lack of effective treatments, the identification of pathways that may provide novel therapeutic targets is absolutely necessary. This review will focus on the involvement of circulating fibrocytes in pulmonary fibrosis.

\section{Pro-fibrotic role of circulating fibrocytes}

The "fibrocyte" was firstly described in 1994 as a circulating, bone marrow-derived cell with the ability to adopt a mesenchymal phenotype, and display an adherent, spindle-shaped, fibroblast-like morphology when cultured in vitro. ${ }^{3}$ Fibrocytes comprise $0.1-1 \%$ of the nucleated cells in the peripheral blood of healthy hosts. ${ }^{4}$ These cells express markers of both hematopoietic cells (CD34, CD45, MHCII) and stromal cells (collagensI/III, fibronectin). ${ }^{5}$ Fibrocytes are rare in normal healthy tissue but are readily detected in fibrotic tissues including those involved the lungs ${ }^{4}$, liver ${ }^{6,7}$, kidney $^{8}$, autoimmunity ${ }^{9,10}$ and skin ${ }^{11}$.

Fibrocytes in the lung tissues and circulation were increased in both aninmal ${ }^{12,13}$ and human pulmonary fibrosis ${ }^{14}$. Although fibrocytes are relatively small in number, these cells could traffic into injured tissue and play an important role in the process of tissue repair and fibrogenesis. Fibrocytes secrete a variety of inflammatory cytokines (IL-13, TGF- $\beta$, CTGF and TNF- $\alpha$, etc.) to augment the fibrotic lesions by activating resident fibroblasts. ${ }^{12,15,16}$ Additionally, fibrocytes acted as a regulator that promoted progressive accumulation of Wilms' Tumor 1 (WT1)-positive cells, a sizable subset of lung-resident mesenchymal cells, in fibrotic lesions of both human idiopathic pulmonary fibrosis (IPF) and mouse models of pulmonary fibrosis. ${ }^{17}$ The circulating fibrocytes secrete periostin, a matricellular 
pro-fibrotic protein that could be upregulated by TGF- $\beta$, thus promote pulmonary fibrogenisis and ECM deposition. ${ }^{18}$ Intrapulmonary TGF- $\beta 1$-induced apoptosis recruited the fibrocytes accumulation and induced collagen production, which was attenuated through apoptosis inhibition. ${ }^{19}$

Fibrocytes could be a source of ECM components that restore the architecture of the damaged tissue and promote angiogenesis and wound contraction at sites of tissue injury. ${ }^{20,21}$ Fibrocyte can be recruited into the bronchial or lung tissue and transform into fibroblast or a-smooth muscle actin (a-SMA) ${ }^{+}$ myofibroblasts, ${ }^{22-24}$ which are the main ECM-producing cells during the pulmonary fibrosis. More than $27 \%$ of the fibrocytes in bleomycin-induced fibrotic lung tissue expressed type I collagen (Col I). These cells constitute more than $80 \%$ of all Col I-expressing cells in the fibrotic lung. ${ }^{24}$ Interestingly, fibrocytes were present in lung tissues but are absent in bronchoalveolar lavage (BAL) of patient with IPF. ${ }^{14}$ The transformation of fibrocytes into fibroblast or myofibroblast was regulated by TGF- $\beta 1$ assisted with semaphorin $7 \mathrm{a}^{9,25,26}$ or endothelin-1 (ET-1). ${ }^{27}$ Th17-derived cytokines IL-17A can induce asthmatic fibrocytes to proliferate and acquire proinflammatory phenotype with increased expression of a-SMA gene and protein. ${ }^{28}$ Fibrocytes lose cell surface phenotype and chemokine receptors such as CD34, CD45, CCR2, CXCR4 during their transformation process, 13, 22 which may serve to trap the transformed fibroblasts and myofibroblasts within the lung and prevent their migration out of the injured lung.

Recently, it has been recognized that deregulated vascular remodeling is one of the most critical biological processes of pulmonary fibrosis. The increased circulating and intrapulmonary fibrocytes secreted pro-angiogenic factors (VEGF, PDGF, b-FGF, HGF, GM-CSF, CTGF) that promote an angiogenic phenotype in endothelial cells.29,30. Moreover, fibrocytes may ease cell migration and further facilitate the angiogenesis process by secreting a variety of matrix metalloproteinases (MMPs). ${ }^{31}$

\section{Differentiation regulation of circulating fibrocyte}

The exact origin of fibrocytes was not identified clearly so far, most studies revealed that fibrocytes were differentiated from a subpopulation of $\mathrm{CD} 14^{+}$ peripheral blood monocytes. ${ }^{32,33}$ Circulating monocytes from patients with IPF and connective tissue disease interstitial lung disease (CTD-ILD) displayed enhanced differentiation into fibrocytes compared with normal healthy controls. ${ }^{19}$ Different culture condition such as blood collection, substrates, media, and cell density all influence in vitro fibrocytes differentiation. ${ }^{33}$ The differentiation of monocytes into fibrocytes was affected by resident fibroblasts. TNF-a-stimulated fibroblasts secreted lumican (a small leucine-rich proteoglycan), one of the main component of ECM, and lumican potentiated fibrocytes differentiation via a2 $\beta 1, \mathrm{aM} \beta 2$, and $\mathrm{aX} \beta 2$ integrins $^{34}$. Whereas, the neuronal guidance protein Slit2 secreted by fibroblast inhibited human fibrocytes differentiation. ${ }^{35}$

Niedermeier and colleagues reported that differentiation of fibrocytes was critically dependent on $\mathrm{CD}^{+} \mathrm{T}$ cells. In the model of unilateral ureteral obstructionthe (UUO) in mice, different way of $\mathrm{T}$ cell activation determines whether development of fibrocytes is supported or blocked. Polyclonal activation of $\mathrm{CD}^{+} \mathrm{T}$ cells induced the release of soluble factors (IL-2, TNF, IFN- $\gamma$ and IL-4), and that completely prevented the outgrowth of fibrocytes and reduced the severity of fibrosis. In contrast, activation of $\mathrm{CD}^{+} \mathrm{T}$ cells in the presence of calcineurin inhibitors (Cyclosporine A) markedly enhanced the outgrowth of fibrocytes and renal deposition of collagen I. ${ }^{36}$ It was found that IL-4 and IL-13 promote fibrocyte differentiation, whereas IL-12 and IFN- $\gamma$ inhibit fibrocyte differentiation. These cytokines have different ways to effect monocyte-to-fibrocyte differentiation. IL-4, IL-13 and IFN- $\gamma$ appear to act directly on monocytes, whereas IL-12 acts indirectly, possibly through NK cells. IL-4 and IL-13 increased the number of precursor cells to differentiate into fibrocytes rather than inducing fibrocyte proliferation, as well as without altering the morphology or phenotype of these cells. ${ }^{37}$ Pilling et al. reported that only heat-aggregated IgG but not monomeric IgG or monomeric or heat-aggregated $\operatorname{IgA}$, IgE or IgM could inhibit fibrocyte differentiation, which suggest that ligation and cross-linking of $\mathrm{Fc}_{\mathrm{C}} \mathrm{Rs}$ are inhibitory signals for monocyte-to-fibrocyte differentiation. ${ }^{38}$ In addition, fibrocyte differentiation could be mediated by epidermal growth factor receptor (EGFR) pathway, 39 lumican,34 low molecular weight hyaluronic acid (LMWHA), ${ }^{40}$ Toll-like receptor 2 (TLR2) agonists. ${ }^{41}$

\section{CXCL12/CXCR4 axis mediated fibrocyte trafficing in pulmonary fibrosis}

Human fibrocytes express several chemokine receptors, including CCR2, CCR5, CCR7, and CXCR4. Fibrocyte traffic into wound site in response to chemokines such as CXCL12, CCL21. Among those mentioned above, the CXCL12/CXCR4 biological axis is the most important one in mediating fibrocyte influx to the fibrotic lung, and this ligand/receptor 
has received a great deal of attention in this regard. ${ }^{4,42}$ CXCR4 is an important chemokine receptor in cell trafficking, and the differential expression of its ligand CXCL12, also known as stromal cell-derived factor 1 (SDF-1), in tissues creates the chemotactic gradient required for trafficking of $\mathrm{CXCR}^{+}$cells. CXCR4 are widely expressed in hematopoietic progenitor cells, white blood cells, lungs, and liver. ${ }^{43,44}$ The binding of CXCL12 to CXCR4 are able to activate various downstream signaling that promote cell proliferation, migration, and fibrotic lesions. The downstream intracellular signaling transduction pathways including Phosphatidylinositol 3-kinase (PI3K)/Akt/ mammalian target of rapamycin (mTOR), Rac1/extracellular signal-regulated kinase (ERK), mitogen-activated protein kinase (MAPK), p38MAPK, stress-activated protein kinase (SAPK), c-Jun N-terminal kinase (JNK)/ activator protein-1 (AP-1), Janus kinase/signal transducers and activators of transcription (JAK/STAT). ${ }^{45,46}$

CXCR4 is the predominant chemokine receptor on human fibrocytes. More and more evidence have indicated that substantial numbers of $\mathrm{CXCR}^{+}$ fibrocytes can migrate and accumulate in lung in response to CXCL12 during pulmonary fibrosis. ${ }^{24}$, ${ }^{47}$ CXCR4 levels increase in the lungs after intratracheal bleomycin, and the synthetic specific CXCR4 antagonist TN14003 attenuated bleomycin-induced pulmonary fibrosis in mice. ${ }^{48}$ CXCR4 expression is upregulated by hypoxia inducible factor-1a (HIF-1 $\alpha$ ), vascular endothelial growth factor(VEGF), PDGF, NF-kB, Wnt/ $\beta$-catenin, bradykinin, IL-1 $\beta$, TNF- $\alpha$, IFN- $\gamma$, TGF- $\beta 1$ and drastically downregulated by the Th2 cytokines IL-5 and IL-4, IL-3, GM-CSF in inflammation or hypoxia conditions. ${ }^{42,}$, $9-54$ It should be noticed that treatment with IL-5, but not IL-4, apparently decreased the level of CXCR4 mRNA in eosinophils, although both cytokines suppressed surface protein expression of CXCR4. It might be possible that each cytokine may regulate CXCR4 expression via different pathways or mechanisms, and surface CXCR4 expression is modulated not only at the level of post-transcription but also at least in part at the level of transcription. ${ }^{50}$ Both hypoxia-induced and PDGF-induced CXCR4 expressions could be attenuated by specific inhibition of PI3 kinase and mTOR. ${ }^{42}$

Phillips et al. firstly reported circulating fibrocytes migrated in response to CXCL12 and trafficked into the lungs in a murine model of bleomycin-induced pulmonary fibrosis, and specific neutralization of CXCL12 inhibited intrapulmonary recruitment of fibrocytes and attenuated lung fibrosis. ${ }^{13}$ The expression of CXCL12 increased significantly under ischemia and hypoxia conditions. ${ }^{55,56}$ The CXCL12 levels in the lungs, bronchoalveolar lavage and serum were found significantly elevated associated with increased circulating fibrocytes in mice and human pulmonary fibrosis. ${ }^{13,42,48,57}$ Rapamycin treatment resulted in a $50 \%$ decrease of CXCL12 expression in lungs. ${ }^{42}$ Regulatory T cells (Tregs) could reduce lung fibrocyte recruitment by inhibiting the CXCL12/CXCR4 axis after acute lung injury. ${ }^{58}$

\section{Clinical pathogenic significance of circulating fibrocyte}

Researches from patients showed that circulating fibrocyte increased in various lung disease including chronic obstructive asthma ${ }^{25}$ acute asthma exacerbation without airflow obstruction ${ }^{59}$, acute exacerbation of chronic obstructive pulmonary disease (AECOPD) ${ }^{60}$, Fibrotic interstitial lung diseases ${ }^{57}$, IPF 61, AECOPD $^{60}$ chronic hypersensitivity pneumonitis ${ }^{62}$, obliterative bronchiolitis $(\mathrm{OB})^{63}$, cystic fibrosis patients ${ }^{64}$, rheumatoid arthritis-usual interstitial pneumonia65, pulmonary hypertension $(\mathrm{PE})^{66}$, sickle cell lung disease ${ }^{67}$. Blood fibrocytes are recruited in lungs during COPD exacerbations and related to mortality and low lung function ${ }^{60}$. The total, activated, and differentiated fibrocytes were increased in asthmatic patients experiencing an asthma exacerbation in the preceding 12 months. The a-SMA ${ }^{+}$fibrocyte was able to differentiate into myofibroblast thus contribute to the airway remodel and pulmonary fibrosis 68 .

The higher quantity of circulating fibrocytes indicated a poor prognosis in IPF61, and increased mortality in patients with acute respiratory distress syndrome (ARDS) ${ }^{69}$. The counts of circulating fibrocytes were significantly elevated in the blood of patients with stable IPF $(2.72 \pm 0.34 \%)$ compared with healthy control subjects $(1 \pm 0.12 \%)$. During episodes of acute exacerbation, fibrocyte counts increased substantially up to 10 -fold above the values measured during stable disease $(14.51 \pm 2.53 \%)^{61}$. Fibrocyte numbers were not correlated with lung function or radiologic severity scores, but they were an independent predictor of early mortality. The mean survival of patients with fibrocytes higher than $5 \%$ of total blood leukocytes was 7.5 months compared with 27 months for patients with less than $5 \%{ }^{61}$. Fujiwara et al. further confirmed a correlation between increased numbers of circulating fibrocytes and activity and progression of interstitial lung diseases (ILD). The number of circulating fibrocytes was significantly and inversely correlated with measurable clinical lung functions parameters including vital capacity (VC) 
and diffusing capacity of the lung for carbon monoxide/alveolar ventilation (DLco/VA), and positively correlated with serum levels of sialylated carbohydrate antigen (KL-6, an inflammatory biomarker of disease activity $)^{70}$. In addition, there are also positive correlation between the amount of circulating fibrocytes and severity of $\mathrm{COPD}^{71}$, asthma ${ }^{68}$, and bronchiolitis obliterans syndrome (BOS) development in lung transplant patients ${ }^{72}$. Taken together, these studies indicated that fibrocytes may be used as an indicator or a clinical biomarker for respiratory fibrogenic activity, disease progression, and prognosis.

\section{Potential therapy targeted on circulating fibrocyte and CXCR4}

Based on these findings that CXCL12/CXCR4 axis mediated recruitment of fibrocytes served as a main pathogenic resource of pulmonary fibrosis. The promising therapeutic targets of fibrocytes differentiation or CXCR4 have received considerable attention. Several small molecule CXCR4 antagonists (e.g. AMD3100, MSX-122, TN14003) have been developed and some of them significantly reduced bleomycin or radiation-induced pulmonary fibrosis in mice $^{48,73-75}$. Plerixafor (solution of AMD3100 for subcutaneous injection, brand name: Mozobil ${ }^{\mathrm{TM}}$, Genzyme Corporation) has been approved by the United States Food and Drug Administration (FDA) and European Medicines Agency (EMA) for use in hematopoietic stem cells (HSC) mobilization for autologous transplant for patients with non-Hodgkin's lymphoma and multiple myeloma $(\mathrm{MM})^{76}$. So, it is also worth to perform clinical trial to evaluate the therapeutically potential of Plerixafor in pulmonary fibrosis patient population in future.

Another selective and potent antagonist of CXCR4 which developed by the protein epitope mimetics technology, named protein epitope mimetic (PEM) POL5551, could inhibit vascular accumulation of CXCR4-expressing smooth muscle cells ${ }^{77}$. Compared with Plerixafor, POL5551 showed a superior ability to block CXCL12-induced responses and mobilize hematopoietic stem and progenitor cells more effectively ${ }^{78}$. In clinical trials, a new small molecule AMD11070 has been reported as an orally bioavailable inhibitor of CXCR4 with safe, well-tolerated, and more favorable pharmacokinetic profile when administered to healthy volunteers ${ }^{79,} 80$. A serial of novel small molecule modulators of the CXCR4 receptor have been developed these years. These new compounds may potentially open up new therapeutics for CXCR4 related pulmonary firbrosis 81,82 .
Serum amyloid $P$ (SAP) is a highly phylogenetically conserved, naturally circulating plasma protein in human being, which has been proven to inhibit the monocyte-to-fibrocyte differentiation as an endogenous inhibitor by binding to FcyRs. Preclinical cell and animal experiments suggested that SAP could reduce bleomycin-induced lung fibrosis ${ }^{38,} 83-85$. Intravenous administration of recombinant form of human SAP (PRM-151) resulted in a $30-50 \%$ decrease in fibrocyte numbers $24 \mathrm{~h}$ post-dose in pulmonary fibrosis patients ${ }^{86}$. This first-in-class modulator of the fibrosis pathway has been granted orphan drug designation for myelofibrosis by FDA, and the phase 2 clinical trials ongoing (NCT01981850) ${ }^{87}$. A phase $1 \mathrm{~b}$ study of intravenous (IV) PRM151 in patients with IPF has been completed and it has advanced to phase 2 clinical testing (NCT01254409). These studies showed that PRM-151 was well tolerated at all dose levels, with no serious adverse reactions. Some clinical efficacy markers including pulmonary function tests, George's Respiratory Questionnaire (SGRQ), blood biomarkers (multiple matrix metalloproteinases, cytokines and chemoattractants, etc.) showed trends towards improvement in the PRM-151 dose groups ${ }^{88}$.

In summary, some potential agents have definitively demonstrated the efficacy and safety, but the amounts of subjects and population included were limited. More randomized, double-blind, multiple-center clinical research with larger amounts of subjects are needed to support these innovative therapeutic approaches that may one day offer the clinical benefits to all pulmonary fibrosis patients.

\section{Competing Interests}

The authors have declared that no competing interest exists.

\section{References}

1. Scotton CJ, Chambers RC. Molecular targets in pulmonary fibrosis: the myofibroblast in focus. Chest, 2007;132(4):1311-1321.

2. Gharaee-Kermani $\mathrm{M}, \mathrm{Hu} \mathrm{B}, \mathrm{Phan} \mathrm{SH}$, et al. Recent advances in molecular targets and treatment of idiopathic pulmonary fibrosis: focus on TGFbeta signaling and the myofibroblast. Curr Med Chem, 2009;16(11):1400-1417.

3. Bucala R, Spiegel LA, Chesney J, et al. Circulating fibrocytes define a new leukocyte subpopulation that mediates tissue repair. Mol Med, 1994;1(1):71-81.

4. Strieter RM, Keeley EC, Hughes MA, et al. The role of circulating mesenchymal progenitor cells (fibrocytes) in the pathogenesis of pulmonary fibrosis. J Leukoc Biol, 2009;86(5):1111-1118.

5. Herzog EL, Bucala R. Fibrocytes in health and disease. Exp Hematol, 2010;38(7):548-556

6. $\mathrm{Xu}$ J, Cong M, Park TJ, et al. Contribution of bone marrow-derived fibrocytes to liver fibrosis. Hepatobiliary Surg Nutr, 2015;4(1):34-47.

7. Scholten D, Reichart D, Paik YH, et al. Migration of fibrocytes in fibrogenic liver injury. Am J Pathol, 2011;179(1):189-198.

8. Wada T, Sakai N, Sakai Y, et al. Involvement of bone-marrow-derived cells in kidney fibrosis. Clin Exp Nephrol, 2011;15(1):8-13.

9. Gan Y, Reilkoff R, Peng X, et al. Role of semaphorin 7a signaling in transforming growth factor beta1-induced lung fibrosis and scleroderma-related interstitial lung disease. Arthritis Rheum, 2011;63(8):2484-2494. 
10. Mathai SK, Gulati M, Peng X, et al. Circulating monocytes from systemic sclerosis patients with interstitial lung disease show an enhanced profibrotic phenotype. Lab Invest, 2010;90(6):812-823.

11. Curran TA, Ghahary A. Evidence of a role for fibrocyte and keratinocyte-like cells in the formation of hypertrophic scars. J Burn Care Res, 2012;34(2):227-231.

12. Madala SK, Edukulla R, Schmidt S, et al. Bone marrow-derived stromal cells are invasive and hyperproliferative and alter transforming growth factor-alpha-induced pulmonary fibrosis. Am J Respir Cell Mol Biol, 2014;50(4):777-786.

13. Phillips RJ, Burdick MD, Hong K, et al. Circulating fibrocytes traffic to the lungs in response to CXCL12 and mediate fibrosis. J Clin Invest, 2004;114(3):438-446.

14. Andersson-Sjoland A, de Alba CG, Nihlberg K, et al. Fibrocytes are a potential source of lung fibroblasts in idiopathic pulmonary fibrosis. Int J Biochem Cell Biol, 2008;40(10):2129-2140.

15. Wang JF, Jiao $\mathrm{H}$, Stewart TL, et al. Fibrocytes from burn patients regulate the activities of fibroblasts. Wound Repair Regen, 2007;15(1):113-121.

16. Chesney J, Metz C, Stavitsky AB, et al. Regulated production of type I collagen and inflammatory cytokines by peripheral blood fibrocytes. J Immunol, 1998;160(1):419-425.

17. Sontake V, Shanmukhappa SK, DiPasquale BA, et al. Fibrocytes Regulate Wilms Tumor 1-Positive Cell Accumulation in Severe Fibrotic Lung Disease. J Immunol, 2015;195(8):3978-3991.

18. Naik PK, Bozyk PD, Bentley JK, et al. Periostin promotes fibrosis and predicts progression in patients with idiopathic pulmonary fibrosis. Am J Physiol Lung Cell Mol Physiol, 2012;303(12):L1046-1056.

19. Peng X, Mathai SK, Murray LA, et al. Local apoptosis promotes collagen production by monocyte-derived cells in transforming growth factor beta1-induced lung fibrosis. Fibrogenesis Tissue Repair, 2011;4(1):1-12.

20. Kleaveland KR, Moore BB, Kim KK. Paracrine functions of fibrocytes to promote lung fibrosis. Expert Rev Respir Med, 2014;8(2):163-172.

21. Loomis-King H, Moore BB. Fibrocytes in the Pathogenesis of Chronic Fibrotic Lung Disease. Curr Respir Med Rev, 2013;9(1):34-41.

22. Moore BB, Kolodsick JE, Thannickal VJ, et al. CCR2-mediated recruitment of fibrocytes to the alveolar space after fibrotic injury. Am J Pathol, 2005;166(3):675-684.

23. Lama VN, Phan SH. The extrapulmonary origin of fibroblasts: stem/progenitor cells and beyond. Proc Am Thorac Soc, 2006;3(4):373-376.

24. Hashimoto $\mathrm{N}$, Jin $\mathrm{H}$, Liu $\mathrm{T}$, et al. Bone marrow-derived progenitor cells in pulmonary fibrosis. J Clin Invest, 2004;113(2):243-252.

25. Wang $\mathrm{CH}$, Huang $\mathrm{CD}$, Lin $\mathrm{HC}$, et al. Increased circulating fibrocytes in asthma with chronic airflow obstruction. Am J Respir Crit Care Med, 2008;178(6):583-591.

26. Schmidt M, Sun G, Stacey MA, et al. Identification of circulating fibrocytes as precursors of bronchial myofibroblasts in asthma. J Immunol, 2003;171(1):380-389.

27. Weng $\mathrm{CM}$, Chen $\mathrm{BC}$, Wang $\mathrm{CH}$, et al. The endothelin A receptor mediates fibrocyte differentiation in chronic obstructive asthma. The involvement of connective tissue growth factor. Am J Respir Crit Care Med, 2013;188(3):298-308

28. Bellini A, Marini MA, Bianchetti L, et al. Interleukin (IL)-4, IL-13, and IL-17A differentially affect the profibrotic and proinflammatory functions of fibrocytes from asthmatic patients. Mucosal Immunol, 2012;5(2):140-149.

29. Frid MG, Brunetti JA, Burke DL, et al. Hypoxia-induced pulmonary vascular remodeling requires recruitment of circulating mesenchymal precursors of a monocyte/macrophage lineage. Am J Pathol, 2006;168(2):659-669.

30. Hartlapp I, Abe R, Saeed RW, et al. Fibrocytes induce an angiogenic phenotype in cultured endothelial cells and promote angiogenesis in vivo. FASEB J, 2001;15(12):2215-2224.

31. Garcia-de-Alba C, Becerril C, Ruiz V, et al. Expression of matrix metalloproteases by fibrocytes: possible role in migration and homing. Am J Respir Crit Care Med, 2010;182(9):1144-1152.

32. Kuwana M, Okazaki $\mathrm{Y}$, Kodama $\mathrm{H}$, et al. Human circulating CD14+ monocytes as a source of progenitors that exhibit mesenchymal cell differentiation. J Leukoc Biol, 2003;74(5):833-845.

33. Pilling D, Vakil V, Gomer RH. Improved serum-free culture conditions for the differentiation of human and murine fibrocytes. J Immunol Methods, 2009;351(1-2):62-70.

34. Pilling D, Vakil V, Cox N, et al. TNF-alpha-stimulated fibroblasts secrete lumican to promote fibrocyte differentiation. Proc Natl Acad Sci U S A, 2015;112(38):11929-11934.

35. Pilling D, Zheng Z, Vakil V, et al. Fibroblasts secrete Slit2 to inhibit fibrocyte differentiation and fibrosis. Proc Natl Acad Sci U S A, 2014;111(51):18291-18296.

36. Niedermeier M, Reich B, Rodriguez Gomez M, et al. CD4+ T cells control the differentiation of Gr1+ monocytes into fibrocytes. Proc Natl Acad Sci U S A, 2009;106(42):17892-17897.

37. Shao DD, Suresh R, Vakil V, et al. Pivotal Advance: Th-1 cytokines inhibit, and Th-2 cytokines promote fibrocyte differentiation. J Leukoc Biol, 2008;83(6):1323-1333.

38. Pilling D, Tucker NM, Gomer RH. Aggregated IgG inhibits the differentiation of human fibrocytes. J Leukoc Biol, 2006;79(6):1242-1251.
39. Wang $\mathrm{CH}$, Huang $\mathrm{CD}$, Lin $\mathrm{HC}$, et al. Increased activation of fibrocytes in patients with chronic obstructive asthma through an epidermal growth factor receptor-dependent pathway. J Allergy Clin Immunol, 2012;129(5):1367-1376.

40. Maharjan AS, Pilling D, Gomer RH. High and low molecular weight hyaluronic acid differentially regulate human fibrocyte differentiation. PLoS One, 2011;6(10):e26078.

41. Maharjan AS, Pilling D, Gomer RH. Toll-like receptor 2 agonists inhibit human fibrocyte differentiation. Fibrogenesis Tissue Repair, 2010;3(23):1-13.

42. Mehrad B, Burdick MD, Strieter RM. Fibrocyte CXCR4 regulation as a therapeutic target in pulmonary fibrosis. Int $\mathrm{J}$ Biochem Cell Biol, 2009;41(8-9):1708-1718

43. Muller A, Homey B, Soto $\mathrm{H}$, et al. Involvement of chemokine receptors in breast cancer metastasis. Nature, 2001;410(6824):50-56.

44. Nagasawa T, Kikutani H, Kishimoto T. Molecular cloning and structure of a pre-B-cell growth-stimulating factor. Proc Natl Acad Sci U S A, 1994;91(6):2305-2309.

45. Singh AK, Arya RK, Trivedi AK, et al. Chemokine receptor trio: CXCR3, CXCR4 and CXCR7 crosstalk via CXCL11 and CXCL12. Cytokine Growth Factor Rev, 2012;24(1):41-49.

46. Lin $\mathrm{CH}$, Shih $\mathrm{CH}$, Tseng $\mathrm{CC}$, et al. CXCL12 induces connective tissue growth factor expression in human lung fibroblasts through the Rac1/ERK, JNK, and AP-1 pathways. PLoS One, 2014;9(8):e104746-e104761.

47. Nakashima T, Liu T, Yu H, et al. Lung bone marrow-derived hematopoietic progenitor cells enhance pulmonary fibrosis. Am J Respir Crit Care Med, 2013;188(8):976-984.

48. Xu J, Mora A, Shim H, et al. Role of the SDF-1/CXCR4 axis in the pathogenesis of lung injury and fibrosis. Am J Respir Cell Mol Biol, 2007;37(3):291-299.

49. Eddleston J, Christiansen SC, Zuraw BL. Functional expression of the C-X-C chemokine receptor CXCR4 by human bronchial epithelial cells: regulation by proinflammatory mediators. J Immunol, 2002;169(11):6445-6451.

50. Nagase H, Miyamasu M, Yamaguchi $M$, et al. Expression of CXCR4 in eosinophils: functional analyses and cytokine-mediated regulation. J Immunol, 2000;164(11):5935-5943.

51. Choe Y, Pleasure SJ. Wnt signaling regulates intermediate precursor production in the postnatal dentate gyrus by regulating CXCR4 expression. Dev Neurosci, 2012;34(6):502-514.

52. Esencay M, Newcomb EW, Zagzag D. HGF upregulates CXCR4 expression in gliomas via NF-kappaB: implications for glioma cell migration. J Neurooncol, 2010;99(1):33-40

53. Zagzag D, Lukyanov $Y$, Lan L, et al. Hypoxia-inducible factor 1 and VEGF upregulate CXCR4 in glioblastoma: implications for angiogenesis and glioma cell invasion. Lab Invest, 2006;86(12):1221-1232.

54. Phillips RJ, Mestas J, Gharaee-Kermani M, et al. Epidermal growth factor and hypoxia-induced expression of CXC chemokine receptor 4 on non-small cell lung cancer cells is regulated by the phosphatidylinositol 3-kinase/PTEN/AKT/mammalian target of rapamycin signaling pathway and activation of hypoxia inducible factor-1alpha. J Biol Chem, 2005;280(23):22473-22481.

55. Ceradini DJ, Kulkarni AR, Callaghan MJ, et al. Progenitor cell trafficking is regulated by hypoxic gradients through HIF-1 induction of SDF-1. Nat Med, 2004;10(8):858-864.

56. Wang YG, Haider HK, Ahmad $\mathrm{N}$, et al. Evidence for ischemia induced host-derived bone marrow cell mobilization into cardiac allografts. Journal of Molecular and Cellular Cardiology, 2006;41(3):478-487.

57. Mehrad B, Burdick MD, Zisman DA, et al. Circulating peripheral blood fibrocytes in human fibrotic interstitial lung disease. Biochem Biophys Res Commun, 2007;353(1):104-108.

58. Garibaldi BT, D'Alessio FR, Mock JR, et al. Regulatory T cells reduce acute lung injury fibroproliferation by decreasing fibrocyte recruitment. Am J Respir Cell Mol Biol, 2013;48(1):35-43.

59. Wang $\mathrm{CH}$, Punde $\mathrm{TH}$, Huang $\mathrm{CD}$, et al. Fibrocyte trafficking in patients with chronic obstructive asthma and during an acute asthma exacerbation. J Allergy Clin Immunol, 2015;135(5):1154-1162.

60. Dupin I, Allard B, Ozier A, et al. Blood fibrocytes are recruited during acute exacerbations of chronic obstructive pulmonary disease through a CXCR4-dependent pathway. J Allergy Clin Immunol, 2016;137(4):1036-1042.

61. Moeller A, Gilpin SE, Ask K, et al. Circulating fibrocytes are an indicator of poor prognosis in idiopathic pulmonary fibrosis. Am J Respir Crit Care Med, 2009;179(7):588-594

62. Garcia de Alba C, Buendia-Roldan I, Salgado A, et al. Fibrocytes contribute to inflammation and fibrosis in chronic hypersensitivity pneumonitis through paracrine effects. Am J Respir Crit Care Med, 2015;191(4):427-436.

63. Andersson-Sjoland A, Erjefalt JS, Bjermer L, et al. Fibrocytes are associated with vascular and parenchymal remodelling in patients with obliterative bronchiolitis. Respir Res, 2009;10(103):1-11.

64. Gilpin SE, Lung K, de Couto GT, et al. Bone marrow-derived progenitor cells in end-stage lung disease patients. BMC Pulm Med, 2013;13(48):1-12

65. Margaritopoulos GA, Antoniou KM, Lasithiotaki I, et al. Expression of SDF-1/CXCR4 axis in bone marrow mesenchymal stem cells derived from rheumatoid arthritis-usual interstitial pneumonia. Clin Exp Rheumatol, 2013;31(4):610-611. 
66. Yeager ME, Nguyen CM, Belchenko DD, et al. Circulating fibrocytes are increased in children and young adults with pulmonary hypertension. Eur Respir J, 2012;39(1):104-111.

67. Field JJ, Burdick MD, DeBaun MR, et al. The role of fibrocytes in sickle cell lung disease. PLoS One, 2012;7(3):e33702.

68. Shipe R, Burdick MD, Strieter BA, et al. Number, activation, and differentiation of circulating fibrocytes correlate with asthma severity. J Allergy Clin Immunol, 2016;137(3):750-757.

69. Quesnel C, Piednoir P, Gelly J, et al. Alveolar fibrocyte percentage is an independent predictor of poor outcome in patients with acute lung injury. Crit Care Med, 2011;40(1):21-28

70. Fujiwara A, Kobayashi H, Masuya M, et al. Correlation between circulating fibrocytes, and activity and progression of interstitial lung diseases. Respirology, 2012;17(4):693-698.

71. Kaddah S, Selim S, Rashed L, et al. Circulating fibrocytes are an indicator of severity and exacerbation in chronic obstructive pulmonary disease. Egyptian Journal of Chest Diseases and Tuberculosis, 2014;63(4):805-813.

72. LaPar DJ, Burdick MD, Emaminia A, et al. Circulating fibrocytes correlate with bronchiolitis obliterans syndrome development after lung transplantation: a novel clinical biomarker. Ann Thorac Surg, 2011:92(2):470-477.

73. Makino H, Aono Y, Azuma M, et al. Antifibrotic effects of CXCR4 antagonist in bleomycin-induced pulmonary fibrosis in mice. J Med Invest, 2013;60(1-2):127-137.

74. Liang Z, Zhan W, Zhu A, et al. Development of a unique small molecule modulator of CXCR4. PLoS One, 2012;7(4):e34038-34050.

75. Shu HK, Yoon Y, Hong S, et al. Inhibition of the CXCL12/CXCR4-axis as preventive therapy for radiation-induced pulmonary fibrosis. PLoS One, 2013;8(11):e79768-79776

76. Fruehauf S. Current clinical indications for plerixafor. Transfus Med Hemother, 2013;40(4):246-250.

77. Hamesch K, Subramanian P, Li X, et al. The CXCR4 antagonist POL5551 is equally effective as sirolimus in reducing neointima formation without impairing re-endothelialisation. Thromb Haemost, 2012;107(2):356-368
78. Karpova D, Dauber K, Spohn G, et al. The novel CXCR4 antagonist POL5551 mobilizes hematopoietic stem and progenitor cells with greater efficiency than Plerixafor. Leukemia, 2013;27(12):2322-2331.

79. Stone ND, Dunaway SB, Flexner C, et al. Multiple-dose escalation study of the safety, pharmacokinetics, and biologic activity of oral AMD070, a selective CXCR4 receptor inhibitor, in human subjects. Antimicrob Agents Chemother, 2007;51(7):2351-2358

80. Skerlj RT, Bridger GJ, Kaller A, et al. Discovery of novel small molecule orally bioavailable C-X-C chemokine receptor 4 antagonists that are potent inhibitors of T-tropic (X4) HIV-1 replication. J Med Chem, 2010;53(8):3376-3388.

81. Mishra RK, Shum AK, Platanias LC, et al. Discovery and characterization of novel small-molecule CXCR4 receptor agonists and antagonists. Sci Rep, 2016 6(1):1-9.

82. Gaines T, Camp D, Bai R, et al. Synthesis and evaluation of 2,5 and 2,6 pyridine-based CXCR4 inhibitors. Bioorg Med Chem, 2016;24(21):5052-5060.

83. Pilling D, Roife D, Wang M, et al. Reduction of bleomycin-induced pulmonary fibrosis by serum amyloid P. J Immunol, 2007;179(6):4035-4044.

84. Cox N, Pilling D, Gomer RH. Serum amyloid P: a systemic regulator of the innate immune response. J Leukoc Biol, 2014;96(5):739-743.

85. Pilling D, Crawford JR, Verbeek JS, et al. Inhibition of murine fibrocyte differentiation by cross-linked IgG is dependent on FcgammaRI. J Leukoc Biol, 2014;96(2):275-282

86. Dillingh MR, van den Blink B, Moerland M, et al. Recombinant human serum amyloid $\mathrm{P}$ in healthy volunteers and patients with pulmonary fibrosis. Pulm Pharmacol Ther, 2013;26(6):672-676.

87. [Internet] A Phase 2 Study Of PRM-151 In Subjects With Myelofibrosis. https://clinicaltrialsgov.

88. van den Blink B, Dillingh MR, Ginns LC, et al. Recombinant human pentraxin-2 therapy in patients with idiopathic pulmonary fibrosis: safety, pharmacokinetics and exploratory efficacy. Eur Respir J, 2016;47(3):889-897. 